\title{
Amamentação e utilização de medicamentos: qual a orientação presente nas bulas de anticoncepcionais e anti-infecciosos?
}

\author{
Breastfeeding and drug use: what is the orientation found in drug \\ package inserts of contraceptives and anti-infective agents?
}

Tatiane da Silva Dal Pizzol (https://orcid.org/0000-0002-7566-7745) ${ }^{1}$

Alessandra Nunes Pinto (https://orcid.org/0000-0002-8280-1244) ${ }^{1}$

Marysabel Pinto Telis Silveira (https://orcid.org/0000-0002-6453-8534) ${ }^{2}$

Maria Carolina de Campos Caetano (https://orcid.org/0000-0001-5809-7464) ${ }^{3}$

Camila Giugliani (https://orcid.org/0000-0002-2652-5214) ${ }^{4}$
${ }^{1}$ Faculdade de Farmácia, Universidade Federal do Rio Grande do Sul (UFRGS). Av. Ipiranga 2752, sala 203, Santana. 90610-000 Porto Alegre RS Brasil. tatiane.silva@ufrgs.br ${ }^{2}$ Departamento de Fisiologia e Farmacologia, Instituto de Biologia, Universidade Federal de Pelotas. Pelotas RS Brasil. ${ }^{3}$ Universidade Federal de Ciências da Saúde de Porto Alegre. Porto Alegre RS Brasil.

${ }^{4}$ Faculdade de Medicina, UFRGS. Porto Alegre RS Brasil.

\begin{abstract}
This article aims to evaluate the conformity between drug package inserts (DPIs) and evidence-based bibliographic sources regarding the presence of contraindications to the use of contraceptives and anti-infective agents during breastfeeding. Contraceptive and anti-infectives were selected, according to ATC, with the updated record in the ANVISA and present in the bibliographic sources Breastfeeding and Use of Medicines and Other Substances, Medications and Mother's Milk, LactMed ${ }^{\circledR}$, Micromedex ${ }^{\circledR}$ and UpToDate ${ }^{\circledR}$. Information was extracted from the DPI "Contraindications" and "Warnings and precautions" sections and compared with the information in the bibliographic sources. The contraindication of the drug during breastfeeding was evaluated. Contraindications were found in the DPIs of five (55.5\%) of the nine contraceptives. The contraindication percentage ranged from 0 to $55.5 \%$ among the bibliographic sources, depending on the source. The percentage was $46.3 \%$ in the DPIs, ranging from 0 to $12.9 \%$ in the bibliographic sources for anti-infectives. There is an agreement between the DPIs and the bibliographic sources regarding contraceptives; regarding anti-infectives, the DPIs are more often contraindicated for use during breastfeeding.
\end{abstract}

Key words Breast Feeding, Drug package inserts, Drug utilization, Contraceptives, Anti-infective agents
Resumo O objetivo deste artigo é avaliar a concordância entre bulas e fontes bibliográficas baseadas em evidências científicas quanto à presença de contraindicação do uso de anticoncepcionais e anti-infecciosos durante a amamentação. Foram selecionados medicamentos anticoncepcionais e anti-infecciosos, segundo a ATC, com registro na ANVISA e presentes nas fontes bibliográficas: $\mathrm{Ma}$ nual Amamentação e Uso de Medicamentos e Outras Substâncias, Medications and Mother's Milk, LactMed ${ }^{\circledR}$, Micromedex $^{\circledR}$ e UpToDate ${ }^{\circledR}$. As informações foram extraídas das seções "Contraindicações" e "Advertências e precauções" das bulas e comparadas com as informações das fontes. Foi avaliada a presença de informação contraindicando o uso do medicamento durante a amamentação. Em cinco (55,5\%) dos nove anticoncepcionais foi verificada contraindicação na bula. Entre as fontes bibliográficas, o percentual variou de $0 \%$ a 55,5\%, dependendo da fonte. Para os anti-infecciosos, o percentual de contraindicação foi de $46,3 \%$ na bula, variando de $0 \%$ a $12,9 \%$ nas fontes. Existe concordância entre as bulas e as fontes bibliográficas com relação aos anticoncepcionais; no caso dos anti-infecciosos, as bulas apresentam contraindicação para o uso durante a amamentação com mais frequência.

Palavras-chave Amamentação, Bulas de medicamentos, Uso de medicamentos, Anticoncepcionais, Anti-infecciosos 


\section{Introdução}

A amamentação traz benefícios bem estabelecidos para a saúde do bebê, sendo uma fonte nutricional segura e completa. O impacto da amamentação na saúde da criança (proteção contra infecções respiratórias, diarreia e desenvolvimento futuro de sobrepeso/obesidade e diabetes) ${ }^{1} \mathrm{e}$ da mulher (proteção contra diabetes, câncer de mama e ovário e aumento do intervalo entre partos) $)^{1,2}$ é bem conhecido e demonstrado por evidências contundentes. A Organização Mundial de Saúde (OMS) recomenda a prática da amamentação até os 2 anos ou mais, sendo exclusiva nos primeiros 6 meses de vida da criança ${ }^{3}$. Apesar disso, no Brasil, apenas 37\% das crianças menores de 6 meses são amamentadas exclusivamente, e $32 \%$ seguem sendo alimentadas com leite materno entre 20 e 23 meses de idade ${ }^{4}$.

Durante a amamentação, o uso de medicamentos é uma prática muito comum $^{5-7}$, sendo que as classes terapêuticas mais prescritas são anticoncepcionais, anti-infecciosos, antidepressivos e analgésicos ${ }^{8,9}$. De modo geral, os medicamentos podem influenciar na produção do leite ${ }^{10}$, e podem ser transferidos para o leite, resultando na exposição da criança aos fármacos, podendo ou não apresentar algum dano ao lactente.

A maioria dos estudos que avaliam os efeitos dos medicamentos na amamentação é realizada em modelos animais ${ }^{11}$. Mesmo com a escassez de estudos em nutrizes, a compreensão dos princípios farmacocinéticos e mecanismos de transferência do fármaco para o leite materno pode auxiliar o médico a tomar decisões apropriadas sobre a prescrição de determinado medicamento, mantendo a amamentação enquanto o problema de saúde é tratado ${ }^{8}$. Pode-se minimizar a exposição da criança ao fármaco se a mãe utilizá-lo logo após as mamadas ou durante o sono do bebê, em uma tentativa de evitar que a criança mame durante o pico de concentração plasmática materna. Além disso, o conhecimento atual indica que poucos fármacos são sabidamente prejudiciais à criança quando consumidos pela mãe que amamenta $^{12}$.

Entretanto, a crença de que o uso de medicamentos é incompatível com a amamentação, gerada por uma percepção de risco muitas vezes equivocada e reforçada por informação sem base em evidências, aumenta o risco de desmame precoce ou de não utilização de medicamentos, mesmo quando há indicação precisa ${ }^{5}$. Poucos estudos têm investigado a concordância de informações entre bulas de medicamentos de dife- rentes laboratórios ${ }^{13}$, ou entre bulas e evidências científicas $^{14-16}$.

O objetivo deste estudo foi avaliar a concordância entre bulas e fontes bibliográficas baseadas em evidências científicas quanto à presença de contraindicação do uso de anticoncepcionais e anti-infecciosos durante a amamentação.

\section{Método}

Trata-se de um estudo descritivo, de abordagem quantitativa, complementada por aspectos de natureza qualitativa relativos às características das fontes bibliográficas utilizadas. As classes de medicamentos selecionadas para esta análise foram anti-infecciosos e anticoncepcionais, por se tratarem de medicamentos comumente utilizados durante o período da amamentação ${ }^{8,9}$, e por representarem medicamentos diferentes em termos de duração do uso, agudo e crônico, respectivamente.

Foram incluídos medicamentos anticoncepcionais e anti-infecciosos de bulas padrão destinadas ao profissional da saúde. Foram excluídos medicamentos sem registro ativo na ANVISA ou ausentes em pelo menos uma das fontes bibliográficas usadas nesta análise, descritas adiante.

Primeiro, foram selecionados todos os fármacos indexados pela Classificação Anatomical Therapeutic Chemical (ATC/DDD Index 2018), da $\mathrm{OMS}^{17}$ como anticoncepcionais e anti-infecciosos, independentemente da via de administração. De acordo com a ATC, os fármacos são classificados em grupos e subgrupos terapêuticos, farmacológicos e químicos. Todos os medicamentos presentes na classificação ATC foram rastreados, a partir do nível 1 , que corresponde ao principal grupo anatômico/farmacológico, a fim de localizar anti-infecciosos presentes não apenas no grupo J (anti-infecciosos de uso sistêmico) e no grupo $\mathrm{P}$ (antiparasitários), mas também em outros grupos onde algum medicamento anti-infeccioso pudesse estar presente, isolado ou em associação. Em relação aos anticoncepcionais, foram rastreados todos os medicamentos presentes no subgrupo terapêutico G03 (hormônios sexuais e moduladores do sistema genital), pertencente ao grupo G (sistema genito urinário e hormônios sexuais). A partir dessa relação inicial de fármacos, foram verificados quais possuíam registro ativo na Agência Nacional de Vigilância Sanitária (ANVISA), órgão de regulação e controle sanitário da produção e consumo de medicamentos, incluindo o estabelecimento de padrões de tex- 
to de bulas e sua aprovação. Para obter a informação sobre o registro ativo dos medicamentos, foram consultados os bancos disponibilizados na página eletrônica da ANVISA ${ }^{18}$. Após a confirmação de registro ativo na ANVISA, foram identificados, para cada um dos fármacos listados, os medicamentos de referência na "Lista de Medicamentos de Referência" da ANVISA, e extraídas as bulas padrão do profissional no Bulário Eletrônico da ANVISA ${ }^{19}$. A identificação dos medicamentos com registro ativo na ANVISA e a extração da informação das bulas ocorreram entre março e abril de 2018.

As informações sobre a compatibilidade do uso do medicamento durante a amamentação foram buscadas nas seções de "Contraindicação" ou "Advertências e Precauções" das bulas, que correspondem às partes onde esse tipo de informação deve ser apresentado na bula, de acordo com a Resolução $\mathrm{n}^{\circ}$ 47, da ANVISA ${ }^{20}$.

As informações das bulas de cada medicamento foram comparadas com as informações presentes nas seguintes fontes bibliográficas de referência: Amamentação e Uso de Medicamentos e Outras Substâncias (2a edição), manual publicado pelo Ministério da Saúde ${ }^{21}$, Medications and Mother's Milk (16 ${ }^{\mathrm{a}}$ edição) ${ }^{12}$, Lactmed ${ }^{\circledR 22}$, UpToDate $^{\circledR 23}$ e Micromedex ${ }^{\circledR 24}$. As fontes bibliográficas selecionadas para este estudo foram definidas a partir de estudos anteriores ${ }^{14,25}$ e por consulta a especialistas, além da disponibilidade em nosso meio.

O manual Amamentação e Uso de Medicamentos e outras Substâncias ${ }^{21}$ contém revisão sobre fármacos e outras substâncias transferidas para o leite materno e seus possíveis efeitos no lactente e/ou na lactação, tendo como referência as publicações da Academia Americana de Pediatria (AAP), da OMS, e do livro Medications and Mothers' Milk (edição de 2008). Possui sistema de classificação em três níveis.

O livro Medications and Mother's Milk, do autor T. W. Hale ${ }^{12}$, dispõe de monografias sobre uma ampla variedade de medicamentos e substâncias naturais, incluindo características farmacológicas relevantes, pesquisas primárias, bem como a classificação da AAP. Utiliza sistema de classificação numérica de cinco pontos para cada medicamento, e é atualizado a cada dois anos.

O UptoDate ${ }^{\circledR 23}$ é um recurso online muito utilizado na prática clínica pelos profissionais da saúde, nos ambientes hospitalar e ambulatorial. É considerada uma das fontes mais atualizadas, fazendo um apanhado das evidências disponíveis para apoiar a tomada de decisão do profissional, que levará em conta também a singularidade de cada situação clínica. Não apresenta sistema de classificação, mas sim um resumo descritivo do uso do medicamento durante a amamentação e recomendações de condutas.

O Micromedex ${ }^{\circledR 24}$ é um recurso online constituído de monografias contendo indicações aprovadas pela agência Food and Drug Administration (FDA), usos off-label e informações sobre a segurança do medicamento, entre outras informações. Apresenta classificação própria sobre a segurança de uso dos medicamentos na amamentação, dividida em quatro categorias conforme as evidências disponíveis e/ou consenso de especialistas. Apresenta classificação da AAP.

O LactMed ${ }^{\circledR 22}$ é um recurso online elaborado por painel de especialistas a partir da literatura científica, atualizado mensalmente. Inclui informações sobre os níveis do fármaco no leite materno, efeitos na criança e efeitos sobre a amamentação e leite materno. Não apresenta sistema de classificação, mas sim um resumo descritivo do uso do medicamento durante a amamentação.

O principal desfecho avaliado foi a presença de informação contraindicando o medicamento durante a amamentação ("sim" ou "não"), considerando as especificidades de cada fonte bibliográfica, cujos sistemas de classificação de risco são apresentados no Quadro 1. A informação foi classificada em "sim", quando a informação claramente contraindicava o uso durante a amamentação (ou indicava a suspensão da amamentação durante o uso do medicamento), e "não", nas outras situações. Bulas que apontavam para a incompatibilidade do uso do medicamento com a amamentação, por meio de frases como "devese decidir pela descontinuação da amamentação ou pela interrupção do tratamento", foram classificadas em "sim”. Nas outras situações (uso compatível ou avaliação de risco/benefício), foram classificadas em "não". Conforme já mencionado, o manual do Ministério da Saúde, o livro Medications and Mother's Milk e a base de dados Micromedex $^{\circledR 12,21,24}$ possuem sistema próprio de classificação. Por sua vez, Lactmed ${ }^{\circledR}$ e UpToDate ${ }^{\circledR 22,23}$ trazem as informações de incompatibilidade em forma de texto; nestas fontes, a presença de informação contraindicando o uso do medicamento durante a amamentação foi classificada como "sim" quando o uso era claramente contraindicado e "não" nas outras situações (uso compatível ou avaliação de risco/benefício). No Lactmed ${ }^{\circledR 22}$, quando a base recomendava a utilização de medicamento alternativo, sem mencionar claramente a possibilidade de uso concomitante, o medica- 
Quadro 1. Classificação da compatibilidade de uso dos medicamentos durante a amamentação em cada uma das fontes bibliográficas pesquisadas ${ }^{\mathrm{a}}$.

\begin{tabular}{|c|c|}
\hline $\begin{array}{c}\text { Fonte de } \\
\text { informação }\end{array}$ & Classificação de risco ${ }^{b}$ \\
\hline \multirow{3}{*}{$\begin{array}{l}\text { Manual Técnico } \\
\text { Amamentação } \\
\text { e Uso de } \\
\text { Medicamentos } \\
\text { e Outras } \\
\text { Substâncias, Brasil }\end{array}$} & $\begin{array}{l}\text { 1. Compatível com a amamentação: Fármacos cujo uso é potencialmente seguro durante } \\
\text { a lactação, haja vista não haver relatos de efeitos farmacológicos significativos para o } \\
\text { lactente }\end{array}$ \\
\hline & $\begin{array}{l}\text { 2. Uso criterioso durante a amamentação: Medicamentos cujo uso no período da lactação } \\
\text { depende da avaliação do risco/benefício. Quando utilizados, exigem monitorização clínica } \\
\text { e/ou laboratorial do lactente, devendo ser utilizados durante o menor tempo e na menor } \\
\text { dose possível. Novos medicamentos cuja segurança durante a amamentação ainda não foi } \\
\text { devidamente documentada encontram-se nesta categoria }\end{array}$ \\
\hline & $\begin{array}{l}\text { 3. Uso contraindicado durante a amamentação: Medicamentos que exigem a interrupção } \\
\text { da amamentação, pelas evidências ou risco significativo de efeitos colaterais importantes } \\
\text { no lactente }\end{array}$ \\
\hline \multirow[t]{5}{*}{$\begin{array}{l}\text { Medications and } \\
\text { Mothers' Milk, } \\
\text { Estados Unidos }\end{array}$} & $\begin{array}{l}\text { L1 Compatível: Medicamento que tem sido utilizado por um grande número de mães que } \\
\text { amamentam, sem qualquer aumento observado em efeitos adversos na criança. Estudos } \\
\text { controlados em mulheres que amamentam não demonstram risco ou possibilidade de } \\
\text { danos para a criança; ou o produto não é biodisponível para uma criança }\end{array}$ \\
\hline & $\begin{array}{l}\text { L2 Provavelmente Compatível: Medicamento que tem sido estudado em um número } \\
\text { limitado de mulheres que amamentam sem um aumento dos efeitos adversos na criança. } \\
\text { E/ou, a evidência de um risco demonstrado com o uso deste medicamento em uma } \\
\text { mulher que amamenta é remoto }\end{array}$ \\
\hline & $\begin{array}{l}\text { L3 Provavelmente Compatível: Não há estudos controlados em mulheres que } \\
\text { amamentam; no entanto, o risco de efeitos adversos para a criança amamentada é } \\
\text { possível, ou estudos controlados mostram que efeitos adversos não são graves, apenas } \\
\text { mínimos. Os medicamentos devem ser dados somente se o benefício potencial justificar o } \\
\text { risco potencial para a criança. (Novos medicamentos que não têm absolutamente nenhum } \\
\text { dado publicado são automaticamente classificados nesta categoria, independentemente de } \\
\text { quão seguros eles possam ser) }\end{array}$ \\
\hline & $\begin{array}{l}\text { L4 Possivelmente Perigoso: Não há evidência de risco para o bebê ou para a produção de } \\
\text { leite materno, mas os benefícios provenientes do uso em mães que amamentam podem } \\
\text { ser aceitáveis apesar do risco para a criança (por exemplo, se o medicamento é necessário } \\
\text { em uma situação de risco de vida ou de uma doença grave para os quais as drogas mais } \\
\text { seguras não podem ser utilizadas ou são ineficazes) }\end{array}$ \\
\hline & $\begin{array}{l}\text { L5 Perigoso: O risco de usar o medicamento em mulheres amamentando claramente } \\
\text { supera qualquer possível benefício da amamentação. O medicamento é contraindicado } \\
\text { em mulheres que estão amamentando um bebê }\end{array}$ \\
\hline \multirow[t]{4}{*}{$\begin{array}{l}\text { Micromedex } \\
\text { Estados Unidos }\end{array}$} & $\begin{array}{l}\text { 1. Risco para a criança não pode ser descartado: evidência disponível e/ou o consenso de } \\
\text { peritos são inconclusivos ou inadequados para determinar o risco para a criança quando } \\
\text { o medicamento é usado durante a amamentação. Pesar os benefícios potenciais do } \\
\text { tratamento medicamentoso contra os riscos potenciais antes de se prescrever o fármaco } \\
\text { durante a amamentação }\end{array}$ \\
\hline & $\begin{array}{l}\text { 2. Risco para a criança tem sido demonstrado: Evidência e /ou consenso de peritos } \\
\text { têm demonstrado efeitos de dano infantil quando o medicamento é usado durante a } \\
\text { amamentação. Uma alternativa para este fármaco deverá ser prescrita ou as pacientes } \\
\text { deverão ser aconselhadas a descontinuar o fármaco durante a amamentação }\end{array}$ \\
\hline & $\begin{array}{l}\text { 3. Risco para a criança é mínimo: o nível de evidência e/ou o consenso de peritos sugerem } \\
\text { que esse fármaco possui risco mínimo para o bebê quando usado durante a amamentação }\end{array}$ \\
\hline & $\begin{array}{l}\text { 4. Efeitos sobre o leite são possíveis: Evidência sugere que este fármaco pode alterar a } \\
\text { produção ou composição do leite. Se uma alternativa a este fármaco não for prescrita, } \\
\text { deve-se monitorar o bebê quanto a efeitos adversos e/ou ingestão adequada de leite }\end{array}$ \\
\hline
\end{tabular}

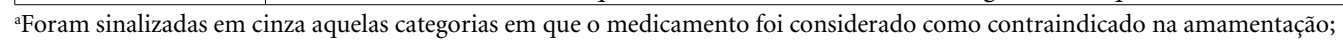
bactMed ${ }^{\circledR}$ e UpToDate ${ }^{\circledR}$ não possuem sistema de classificação. 
mento foi classificado como contraindicado. No UpToDate ${ }^{\circledR 23}$, quando era mencionado que determinada informação provinha do laboratório fabricante, esta foi considerada na classificação do medicamento.

A classificação da informação em "sim" ou "não" foi realizada por dois revisores (ANP e MCC), de forma independente, e as discordâncias foram avaliadas por um terceiro revisor (TSP). O terceiro revisor, farmacêutico, possui experiência em informação sobre medicamentos, tendo coordenado um Centro de Informações sobre Medicamentos por vários anos. Foram analisados apenas os anti-infecciosos e anticoncepcionais hormonais contendo bula padrão e presentes nas cinco fontes bibliográficas. Para cada classe de medicamentos foi verificada a concordância entre as classificações dos dois revisores. Calculou-se o total de medicamentos que receberam a mesma classificação pelos dois revisores, dividido pelo total de medicamentos pertencentes àquela classe, com aplicação do teste de Kappa. Para interpretação do valor encontrado no teste de Kappa, foi utilizado o critério de concordância de Landis e Koch ${ }^{26}$, conforme os valores: $<0,40$, concordância razoável; de 0,41 a 0,60, concordância moderada; de 0,61 a 0,80 , concordância substancial; e de 0,81 a 1,00 , concordância excelente.

As medidas de frequência foram calculadas no programa Excel 2013, e o teste de Kappa foi realizado no programa estatístico SPSS 18.0.0.0 for Windows (SPSS Inc., Chicago, IL, EUA).

\section{Resultados}

No total, foram avaliados 54 anti-infecciosos e nove anticoncepcionais, presentes nas cinco fontes bibliográficas. Foram excluídos os seguintes medicamentos, pela ausência de informação sobre o fármaco em uma ou mais fontes bibliográficas ou ausência de registro no Brasil: benzilpenicilina benzatina (J01CE08), cefalotina (J01DB03), oxitetraciclina (J01AA06), teicoplanina (J01XA02), sulbactam (J01CG01), vancomicina (J01XA01), valaciclovir (J05AB11), secnidazol (P01AB07), antimionato de meglumina (P01CB01), artesunato e mefloquina (P01BF02), levamisol (P02CE01), lincomicina (J01FF02), tiabendazol (P02CA02), ertapenem (J01DH03), meropenem (J01DH02), griseofulvina (D01BA01), sulfadiazina de prata (D06BA01), acetato de clormadinona e etinilestradiol (G03AA15), desogestrel (G03AC09), acetato de medroxiprogesterona e cipionato de estradiol (G03FA12), gestodeno e etinilestradiol (G03AB06), enantanato de noretisterona e valerato de estradiol (G03FA01), etonogestrel e etinilestradiol (G03AC08), acetato de noretisterona e etinilestradiol (G03AA05) e acetato de nomegestrol e estradiol (G03AA14).

A concordância entre os dois revisores (ANP e MCC) na avaliação das bulas foi de $77,8 \%$ para os anticoncepcionais (Kappa 0,564; $\mathrm{p}=0,10$ ) e $85,2 \%$ para os anti-infecciosos (Kappa 0,602; $\mathrm{p}<0,001)$. As discordâncias foram resolvidas por um terceiro revisor (TSP), levando em conta os critérios para classificação em "sim" ou "não".

O Gráfico 1 apresenta a frequência de contraindicação do uso dos medicamentos nas bulas e nas cinco fontes bibliográficas. Foi verificado que $55,5 \%$ das bulas de anticoncepcionais os contraindicavam durante a amamentação. A mesma frequência foi observada em três das cinco fontes avaliadas para esse grupo terapêutico. Em contraste, em uma das outras duas fontes não houve contraindicação. Em relação aos anti-infecciosos, identificou-se contraindicação nas bulas em $46,3 \%$ dos medicamentos, enquanto nas fontes bibliográficas as frequências variaram entre $0 \%$ e $12,9 \%$.

Nas Tabelas 1 e 2 são apresentadas as classificações para cada medicamento avaliado, de acordo com as bulas e as fontes bibliográficas. Em relação aos anticoncepcionais (Tabela 1), foi observada total concordância entre as bulas e as fontes de informação quanto à compatibilidade do uso de preparações contendo progestágenos isolados durante a amamentação. Para os anticoncepcionais que contêm estrógeno associado a progestágeno, houve concordância entre as bulas e o manual do Ministério da Saúde, Lactmed ${ }^{\circledR}$ UptoDate $^{\circledR}$. A fonte Medications and Mothers' Milk não contraindicava o uso de nenhum dos anticoncepcionais analisados.

Para os anti-infecciosos (Tabela 2), foi observada total concordância na não contraindicação, entre as bulas e as fontes, para os seguintes anti-infecciosos: cloranfenicol, amoxicilina, piperacilina, oxacilina, todas as cefalosporinas analisadas, meropenen, sulfametoxazol e trimetropima, eritromicina, claritromicina, gentamicina, norfloxacino, nitrofurantoína, itraconazol, rifampicina, aciclovir, lamivudina, oseltamivir, cloroquina, mebendazol, praziquantel, ivermectina e nistatina. As discordâncias são observadas na contraindicação, pois as bulas contraindicam maior número de anti-infecciosos, com exceção de linezolida e aztreonam, que não apresentam 




Gráfico 1. Frequência de contraindicação de uso de anticoncepcionais e anti-infecciosos durante a amamentação nas bulas e nas fontes bibliográficas ${ }^{a}$.

${ }^{\text {a }}$ Foram avaliadas 77 bulas de anti-infecciosos, correspondendo a 54 medicamentos, e 17 bulas de anticoncepcionais, correspondendo a 9 medicamentos. De duas bulas de azitromicina, uma apresentou contraindicação. De três bulas de tobramicina, uma apresentou contraindicação. Nesses casos, o medicamento foi contabilizado como contraindicado. Manual: Manual Técnico Amamentação e Uso de Medicamentos e Outras Substâncias, do Ministério da Saúde (2010); Hale: Medications and Mothers' Milk, de Hale \& Rowe (2014).

Fonte: Elaborado pelas autoras.

Tabela 1. Classificação dos anticoncepcionais presentes em todas as fontes $(n=9)$ de acordo com a contraindicação de uso durante a amamentação nas bulas dos profissionais e nas fontes bibliográficas.

\begin{tabular}{|c|c|c|c|c|c|c|c|}
\hline $\begin{array}{c}\text { Substância ativa (ATC) } \\
\text { (número de bulas avaliadas) }^{\mathrm{a}}\end{array}$ & ATC & Bula & Manual $^{\text {b }}$ & Hale $^{c}$ & Lactmed $^{\circledR}$ & Micromedex $^{\circledR}$ & Uptodate $^{\circledR}$ \\
\hline \multicolumn{8}{|l|}{$\begin{array}{l}\text { Progestágenos e estrógenos, } \\
\text { combinações a doses fixas (G03AA) }\end{array}$} \\
\hline Levonorgestrel e Etinilestradiol (4) & G03AA07 & $\mathrm{X}$ & $\mathrm{X}$ & & $\mathrm{X}$ & & $\mathrm{X}$ \\
\hline Desogestrel e Etinilestradiol) (4) & G03AA09 & $\mathrm{X}$ & $\mathrm{X}$ & & $\mathrm{X}$ & $\mathrm{X}$ & $\mathrm{X}$ \\
\hline Drospirenona e Etinilestradiol (2) & G03AA12 & $\mathrm{X}$ & $\mathrm{X}$ & & $\mathrm{X}$ & & $\mathrm{X}$ \\
\hline Norelgestromina e Etinilestradiol (1) & G03AA13 & $\mathrm{X}$ & $\mathrm{X}$ & & $\mathrm{X}$ & $\mathrm{X}$ & $\mathrm{X}$ \\
\hline \multicolumn{8}{|l|}{ Progestágenos (G03AC) } \\
\hline Noretisterona (1) & G03AC01 & & & & & & \\
\hline Levonorgestrel (1) & G03AC03 & & & & & & \\
\hline Medroxiprogesterona (2) & G03AC06 & & & & & & \\
\hline Etonogestrel (1) & G03AC08 & & & & & & \\
\hline \multicolumn{8}{|l|}{$\begin{array}{l}\text { Progestágenos e estrógenos, } \\
\text { preparações sequenciais (G03AB) }\end{array}$} \\
\hline Dienogestel e Estradiol (1) & G03AB08 & $\mathrm{X}$ & $\mathrm{X}$ & & $\mathrm{X}$ & & $\mathrm{X}$ \\
\hline
\end{tabular}

contraindicação na bula, mas uma das fontes os contraindica. $\mathrm{O}$ anti-infeccioso que apresentou maior concordância de contraindicação entre as fontes foi o ganciclovir, contraindicado em três das cinco fontes de informação. 
Tabela 2. Classificação dos anti-infecciosos presentes em todas as fontes $(n=54)$ de acordo com a contraindicação de uso durante a amamentação nas bulas dos profissionais e nas fontes avaliadas.

\begin{tabular}{|c|c|c|c|c|c|c|c|}
\hline $\begin{array}{c}\text { Substância ativa } \\
\text { (número de bulas avaliadas) }^{\mathrm{a}}\end{array}$ & ATC & Bula & Manual $^{\mathrm{b}}$ & Hale $^{\mathrm{c}}$ & Lactmed $^{\circledR}$ & Micromedex $^{\circledR}$ & Uptodate $^{\circledR}$ \\
\hline \multicolumn{8}{|l|}{ Antimicrobianos uso sistêmico (J01) } \\
\hline Doxiciclina (1) & J01AA02 & $\mathrm{X}$ & & & & & \\
\hline Minociclina (1) & J01AA08 & $\mathrm{X}$ & & & & & \\
\hline Cloranfenicol (2) & J01BA01 & & & & & & \\
\hline Ampicilina (1) & J01CA01 & $\mathrm{X}$ & & & & & \\
\hline Amoxicilina (2) & J01CA04 & & & & & & \\
\hline Piperacilina (1) & J01CA12 & & & & & & \\
\hline Amoxicilina e Clavulanato (1) & J01CR02 & & & & & & \\
\hline Oxacilina (1) & J01CF04 & & & & & & \\
\hline Cefalexina (3) & J01DB01 & & & & & & \\
\hline Cefazolina (1) & J01DB04 & & & & & & \\
\hline Cefuroxima (1) & J01DC02 & & & & & & \\
\hline Ceftazidima (1) & J01DD02 & & & & & & \\
\hline Ceftriaxona (1) & J01DD04 & & & & & & \\
\hline Cefepima (1) & J01DE01 & & & & & & \\
\hline Aztreonam (1) & J01DF01 & & & & & & $\mathrm{X}$ \\
\hline Meropenen (1) & J01DH02 & & & & & & \\
\hline Imipinem e Cilastatina (1) & J01DH51 & $\mathrm{X}$ & & & & & \\
\hline Sulfametoxazol e Trimetropima (2) & J01EE01 & & & & & & \\
\hline Eritromicina (1) & J01FA01 & & & & & & \\
\hline Claritromicina (2) & J01FA09 & & & & & & \\
\hline Azitromicina $(2)^{\mathrm{d}}$ & J01FA10 & $\mathrm{X}$ & & & & & \\
\hline Clindamicina (3) & J01FF01 & $\mathrm{X}$ & & & & & \\
\hline Tobramicina $(3)^{\mathrm{d}}$ & J01GB01 & $\mathrm{X}$ & & & & & \\
\hline Gentamicina G (3) & J01GB03 & & & & & & \\
\hline Amicacina (1) & J01GB06 & $\mathrm{X}$ & & & & & \\
\hline Ofloxacino (1) & J01MA01 & $\mathrm{X}$ & & & & & \\
\hline Ciprofloxacino (5) & J01MA02 & $\mathrm{X}$ & & & & & \\
\hline Norfloxacino (1) & J01MA06 & & & & & & \\
\hline Levofloxacino (3) & J01MA12 & $\mathrm{X}$ & & & & & \\
\hline Moxifloxacino (2) & J01MA14 & $\mathrm{X}$ & & & & & \\
\hline Daptomicina (1) & J01XX09 & $\mathrm{X}$ & & & & & \\
\hline Linezolida (1) & J01XX08 & & $\mathrm{X}$ & & & & \\
\hline Metronidazol (2) & J01XD01 & $\mathrm{X}$ & & & & & $\mathrm{X}$ \\
\hline Tinidazol (1) & J01XD02 & $\mathrm{X}$ & & & & & $\mathrm{X}$ \\
\hline Nitrofurantoína (1) & J01XE01 & & & & & & \\
\hline Tetraciclina e Anfotericina B (1) & J01AA07 & $\mathrm{X}$ & & & & & \\
\hline
\end{tabular}

\section{Discussão}

Este estudo tem o intuito de auxiliar na tomada de decisões, tanto na prática clínica como na regulação de medicamentos, tendo em vista que a bula pode ser consultada pelo profissional da saúde como fonte de informações sobre a com- patibilidade entre amamentação e o uso do medicamento prescrito.

A cada dois medicamentos avaliados, considerando tanto anti-infecciosos quanto anticoncepcionais, um possuía bula contraindicando o uso durante a amamentação. Para os anti-infecciosos, a concordância entre as bulas e as fontes 
Tabela 2. Classificação dos anti-infecciosos presentes em todas as fontes ( $\mathrm{n}=54)$ de acordo com a contraindicação de uso durante a amamentação nas bulas dos profissionais e nas fontes avaliadas.

\begin{tabular}{|c|c|c|c|c|c|c|c|}
\hline $\begin{array}{c}\text { Substância ativa } \\
\text { (número de bulas avaliadas) }^{\mathrm{a}}\end{array}$ & ATC & Bula & Manual $^{\mathrm{b}}$ & Hale $^{c}$ & Lactmed $^{\circledR}$ & Micromedex $^{\circledR}$ & Uptodate $^{\circledR}$ \\
\hline \multicolumn{8}{|l|}{ Antimicóticos (J02) } \\
\hline Anfotericina B (1) & J02AA01 & $\mathrm{X}$ & & & & & $\mathrm{X}$ \\
\hline Cetoconazol (3) & J02AB02 & $\mathrm{X}$ & & & & & $\mathrm{X}$ \\
\hline Fluconazol (1) & J02AC01 & $\mathrm{X}$ & & & & & \\
\hline Itraconazol (1) & J02AC02 & & & & & & \\
\hline \multicolumn{8}{|l|}{ Antimicobacterianos (J04) } \\
\hline Rifampicina (1) & J04AB02 & & & & & & \\
\hline \multicolumn{8}{|l|}{ Antivirais (J05) } \\
\hline Aciclovir (1) & $\mathrm{J} 05 \mathrm{AB} 01$ & & & & & & \\
\hline Ganciclovir (1) & J05AB06 & $\mathrm{X}$ & $\mathrm{X}$ & & $\mathrm{X}$ & & $\mathrm{X}$ \\
\hline Saquinavir (1) & J05AE01 & $\mathrm{X}$ & & $\mathrm{X}$ & & & \\
\hline Lamivudina (1) & J05AF05 & & & & & & \\
\hline Nevirapina (1) & J05AG01 & $\mathrm{X}$ & & $\mathrm{X}$ & & & \\
\hline Oseltamivir (1) & J05AH02 & & & & & & \\
\hline \multicolumn{8}{|l|}{ Antiprotozoários (P01) } \\
\hline Cloroquina (1) & P01BA01 & & & & & & \\
\hline \multicolumn{8}{|l|}{ Anti-helmínticos (P02) } \\
\hline Albendazol (1) & P02CA03 & $\mathrm{X}$ & & & & & \\
\hline Mebendazol (1) & P02CA01 & & & & & & \\
\hline Praziquantel (1) & P02BA01 & & & & & & \\
\hline Ivermectina (1) & P02CF01 & & & & & & \\
\hline \multicolumn{8}{|l|}{ Diversos } \\
\hline Amantadina (1) & N04BB01 & $\mathrm{X}$ & & & $\mathrm{X}$ & & \\
\hline Nistatina (1) & D01AA01 & & & & & & \\
\hline
\end{tabular}

${ }^{\mathrm{a}}$ Foram avaliadas 77 bulas, correspondendo a 54 medicamentos; ${ }^{\text {b} M a n u a l ~ T e ́ c n i c o ~ A m a m e n t a c ̧ a ̃ o ~ e ~ U s o ~ d e ~ M e d i c a m e n t o s ~ e ~ O u t r a s ~}$ Substâncias, do Ministério da Saúde (2010); 'Medications and Mothers' Milk, de Hale \& Rowe (2014); 'De duas bulas de azitromicina, uma apresentou contraindicação. De três bulas de tobramicina, uma apresentou contraindicação. Nesses casos, o medicamento foi contabilizado como contraindicado. X - Medicamento contraindicado.

Fonte: Elaborado pelas autoras.

foi baixa $(46,3 \%$ das bulas contraindicavam, comparado com $0 \%$ a $12,9 \%$, dependendo da fonte). Para os anticoncepcionais, foi verificada maior concordância na classificação entre as bulas e as fontes.

A identificação de informações inconsistentes nas bulas de medicamentos foi relatada em estudos prévios, seja entre bulas de diferentes laboratórios ${ }^{13}$, ou entre as bulas e as evidências científicas $^{14-16}$. Em análise de 23 bulas de medicamentos antidepressivos e fontes bibliográficas, Dal Pizzol et al. ${ }^{14}$ verificaram que na maioria das bulas $(62,5 \%)$, o antidepressivo foi contraindicado na amamentação, ao passo que, entre as fontes bibliográficas, o percentual variou de $0 \%$ a $25 \%$. Arguello et al. ${ }^{27}$ avaliaram a completude das informações sobre uso durante a gravidez e lactação contidas em bulas europeias. De 534 bulas avaliadas, apenas 16,5\% continham informação de que o fármaco era excretado no leite materno, $0,6 \%$ que não era excretado no leite e $61,4 \%$ afirmaram que esta informação não era conhecida, sendo que $21,5 \%$ das bulas não forneceram qualquer informação relativa à excreção do medicamento no leite. Os autores alertam que, se a agência reguladora considerar a informação insuficiente, mais dados deveriam ser requisitados ao laboratório, durante o processo de autorização ${ }^{27}$. Em conjunto, os estudos citados revelam deficiência de informação a respeito do tema, e que essa situação não ocorre somente no Brasil.

A maior concordância observada no grupo dos anticoncepcionais, em comparação com os anti-infecciosos, já era esperada, em parte porque a variedade de medicamentos analisados foi menor (9 versus 54). Tendo em vista que a con- 
tracepção é um evento mais frequente em comparação às infecções, ao menos entre as nutrizes, é de se esperar que os anticoncepcionais tenham maior potencial de uso em comparação aos anti -infecciosos. Além disso, na maioria dos casos, o uso do anticoncepcional é contínuo e duradouro, diferentemente dos anti-infecciosos, usados predominantemente em condições agudas e por tempo limitado. Esses fatores podem contribuir para maior acúmulo de evidências sobre seus efeitos nesta população.

Os progestágenos combinados aos estrógenos foram contraindicados em todas as bulas e na maior parte das fontes bibliográficas. A contraindicação decorre do potencial dos estrógenos em diminuir a produção de leite, pelo efeito inibidor da prolactina ${ }^{20}$, embora esse efeito seja dependente da dose do medicamento e de cada indivíduo. Porém, chama a atenção o fato da fonte Medications and Mother's Milk não contraindicar o uso de nenhum anticoncepcional durante a amamentação. Esta fonte classifica todas as combinações de anticoncepcionais contendo etinilestradiol em L3 (dados limitados - provavelmente compatíveis), provavelmente porque os efeitos sobre a diminuição da produção de leite são dose dependentes ${ }^{12}$. Dessa forma, na maioria dos casos, a opção tem sido o uso de contraceptivos que contenham apenas progestágeno. De modo geral, a elevada concordância observada entre as bulas e as fontes bibliográficas sugere que as bulas de anticoncepcionais estariam sendo consistentes na orientação quanto ao seu uso durante a amamentação, proporcionando maior segurança e tranquilidade para as nutrizes.

Por outro lado, para os anti-infecciosos, a concordância entre as bulas e as fontes utilizadas foi menor. O elevado número de anti-infecciosos cujas bulas contraindicam o uso na amamentação (aproximadamente uma a cada duas bulas), contrasta com a classificação verificada nas fontes bibliográficas. Poucos anti-infecciosos foram consistentemente contraindicados pelas fontes. O único anti-infecioso com mais fontes classificando-o como contraindicado (três das cinco fontes) foi o ganciclovir.

Quando avaliamos os anti-infecciosos por subgrupo químico (nível 4 da classificação ATC), contendo dois ou mais representantes por subgrupo, chama a atenção a discordância total observada entre as bulas e as fontes para as tetraciclinas e fluorquinolonas. Embora algumas fontes apontem para o uso criterioso das tetracicilinas durante a amamentação, nenhuma as contraindica. O Medications and Mother's Milk, por exem- plo, classifica a doxiciclina e a minociclina em L3 - provavelmente compatível ${ }^{12}$. O manual do Ministério da Saúde aponta uso criterioso para a doxiciclina, alertando para a possiblidade de manchas nos dentes, e uso compatível da minociclina quando utilizada por período menor que três semanas ${ }^{21}$. É possível que a contraindicação nas bulas esteja associada à possível coloração do esmalte dentário ou deposição óssea de tetraciclinas. No entanto, o Lactmed ${ }^{\circledR}$ ressalta que não há probabilidade de haver danos no uso a curto prazo de minociclina e doxiciclina, porque os níveis no leite são baixos e a absorção pela criança é inibida pelo cálcio do leite materno. O uso a curto prazo de minociclina é aceitável em mães que amamentam ${ }^{22}$.

Padrão similar é verificado com as fluorquinolonas. Na base Lactmed ${ }^{\circledR}$, o uso de ciprofloxacina, ofloxacina, levofloxacina e moxifloxacina durante a amamentação é aceitável, com a recomendação de monitorar a criança para possíveis efeitos adversos na flora gastrointestinal (tais como diarreia ou candidíase) e amamentar somente 3 a 6 horas após a administração do medicamento ${ }^{22}$.

Em face do desacordo identificado entre as recomendações das bulas e das fontes para esses dois subgrupos de anti-infecciosos, podemos sugerir que, havendo qualquer risco, mesmo que mínimo ou controlado por um uso criterioso (com uso por tempo limitado ou afastamento dos horários entre a amamentação e a administração do medicamento), as indústrias farmacêuticas optam por contraindicar o uso a diferenciar os níveis do risco e as condições de uso do medicamento durante a amamentação. A veiculação de informação conservadora nas bulas de medicamentos parece refletir um posicionamento de proteção da indústria farmacêutica contra possíveis ações legais, conforme já apontado em estudo anterior ${ }^{14}$. Este posicionamento protecionista da indústria farmacêutica pode ser ilustrado pela presença frequente de frases genéricas, como $A$ decisão entre a descontinuação do medicamento ou da amamentação, deve ser tomada levando-se em consideração os potenciais benefícios do medicamento para a mãe e menção à inexistência de estudos clínicos. Esse posicionamento pode ser questionado, quando as fontes de referência apontam para o uso seguro do medicamento nesse período. Informações limitadas sobre o efeito do medicamento na amamentação podem levar, por um lado, ao desmame precoce ou, por outro lado, à redução ou descontinuação do uso de medicamentos pelas nutrizes; ambos 
com consequências para a saúde da mãe e da criança ${ }^{28}$. Mais que um requisito legal para o registro do produto, as bulas são uma fonte oficial de informações sobre medicamentos, e esse posicionamento defensivo da indústria não contribui para que as bulas informem adequadamente os profissionais da saúde ${ }^{27}$.

Em última análise, as bulas são o principal documento oficial que os profissionais da saúde brasileiros têm para usar como fonte de informação, pois, além da indisponibilidade de um formulário terapêutico atualizado, este não contempla todos os medicamentos comercializados no país, limitando-se aos presentes na Relação Nacional de Medicamentos Essenciais (RENAME). Os livros especializados e demais fontes não brasileiras nem sempre são acessíveis e incluem os fármacos prescritos e usados no Brasil. Assim sendo, existe uma carência de informação rápida, acessível e confiável para o profissional e a bula, que poderia exercer este papel, não o faz adequadamente.

Entre os anti-infecciosos, chama a atenção a alta concordância entre bulas e fontes bibliográficas para penicilinas e cefalosporinas. Esse achado é provavelmente reflexo do maior acúmulo de estudos sobre esses medicamentos, com mais evidências sobre sua segurança. É possível que boa parte da discordância entre bulas e fontes seja consequência da pouca disponibilidade de evidências, principalmente de boa qualidade, sobre fármacos na amamentação. A maioria dos estudos em humanos é observacional, havendo também grande quantidade de relatos de caso. Assim, de forma geral, o nível de evidência para a segurança de fármacos e outras substâncias na gestação e na amamentação é baixo ou muito baixo, contribuindo para a posição conservadora das indústrias ${ }^{29}$.

Alguns aspectos sobre as bases bibliográficas usadas nesta análise merecem destaque. As evidências usadas para a classificação de risco, bem como o próprio sistema de classificação, podem diferir de forma expressiva entre as fontes. Quando existem dúvidas sobre o risco na amamentação, sem evidências suficientes para contraindicar o uso concomitante, o manual do Ministério da Saúde e o LactMed ${ }^{\circledR}$ estimulam a amamentação, respaldados pela literatura. Por outro lado, aquelas fontes que baseiam suas recomendações a partir de dados do fabricante, tais como o UptoDate ${ }^{\circledR}$, contribuem para uma decisão mais conservadora, semelhante às bulas brasileiras ${ }^{14}$. Além disso, algumas fontes são atualizadas com mais frequência do que outras, fato que pode levar a classificações distintas.
A legislação de bulas no Brasil foi sendo desenvolvida ao longo do século XX, em especial a partir da década de 1980, com a publicação de portarias e resoluções que, gradativamente, acrescentaram itens de informação obrigatórios e aprimoraram aspectos relacionados a formatação e linguagem das bulas ${ }^{30,31}$. No entanto, observamos que a informação relativa sobre a compatibilidade do medicamento na lactação, exigida por essas normas, é bastante limitada. Na Portaria 110, de 1997, era exigido apenas a inclusão da frase "não deve ser utilizado durante a gravidez e a lactação", quando for o caso. A RDC 140, de 2003, estabelecia a descrição de advertências e recomendações sobre o uso adequado do medicamento por grupos de risco, entre os quais os lactentes. A RDC 47, em vigência, acrescenta orientações sobre monitoramento e ajuste de dose, quando aplicável. No entanto, informações específicas sobre a excreção do fármaco no leite materno e efeitos adversos no lactente não são exigidas na resolução. Resultados de estudos clínicos ou pré-clinicos, quando existentes, ou mesmo a informação sobre a inexistência desses estudos contribuiriam para a decisão do profissional da saúde em prescrever ou não o medicamento. No entanto, a normativa brasileira também não estabelece esse tipo de informação, exigida, por exemplo, na legislação da Agência Europeia de Medicamentos ${ }^{32}$. Apesar disso, observamos que muitas bulas simplesmente informam sobre se o fármaco é ou não excretado no leite materno, sem incluir informações adicionais necessárias para a tomada de decisão, como, por exemplo, se a quantidade excretada é clinicamente relevante, quais os possíveis danos ao lactente, e quais as evidências disponíveis.

Como uma limitação do estudo, pode-se citar o fato que, como nem todos os medicamentos listados na WHO ATC possuem registro no Brasil, e alguns não estavam presentes em uma ou mais fontes bibliográficas, não foi possível avaliar um maior número de anti-infecciosos e anticoncepcionais. Como não foi estabelecida uma hierarquia entre as fontes bibliográficas, dada as características de cada uma, decidimos excluir o medicamento que estivesse ausente em qualquer uma das fontes, sem diferenciação entre elas. No entanto, como pelo menos um medicamento de cada classe estava registrado no Brasil, acredita-se que os resultados encontrados não seriam muito diferentes, pois a incompatibilidade de uso, na maioria dos casos, estava vinculada com a classe terapêutica e não com o fármaco em si. Outra limitação diz respeito à concordância moderada entre os revisores na classificação das infor- 
mações presentes nas bulas. $\mathrm{O}$ principal motivo para as discordâncias entre os revisores ocorreu com a frase "deve-se decidir pela descontinuação da amamentação ou pela interrupção do tratamento", e variações da mesma, mas contendo a mesma ideia central. Uma das revisoras entendeu como contraindicação e a outra não. No desempate, foi considerado como contraindicação, já que essa informação não dava a possibilidade do uso concomitante.

Em conclusão, esta análise revela que a cada dois medicamentos, um possuía bula contraindicando o uso durante a amamentação, e que existe baixa concordância entre as informações disponíveis nas bulas dos anti-infecciosos avaliados e as fontes de informação consultadas. No caso dos anticoncepcionais, a concordância foi maior. Os dados relativos aos anti-infecciosos, de modo geral, sugerem maior cautela na utilização das informações veiculadas quanto à amamentação presentes em bulas. Antes de qualquer tomada de decisão envolvendo a prescrição e orientação para o uso desses medicamentos em nutrizes, os prescritores devem buscar informações mais confiáveis, em fontes bibliográficas embasadas em evidências científicas atualizadas. Finalmente, recomenda-se uma revisão da Resolução RDC 47 quanto ao conteúdo e formato da informação exigida para as seções de "Contraindicação" ou "Advertências e Precauções" das bulas-padrão. A informação não deve ser limitada à excreção do medicamento no leite materno, indicando se a mesma tem significância clínica e precisando os efeitos que podem ser prejudiciais para o lactente ou para a amamentação, bem como a necessidade de realização de estudos pós comercialização que forneçam evidências científicas sobre esses efeitos.

\section{Colaboradores}

TS Dal Pizzol: delineou o estudo, analisou e interpretou os resultados, escreveu o manuscrito, revisou a literatura e aprovou a versão final do manuscrito. AN Pinto: coletou os dados, revisou a literatura, escreveu o manuscrito e aprovou a versão final do manuscrito. MCC Caetano: coletou os dados, revisou a literatura e aprovou a versão final do manuscrito. MPT Silveira: analisou e interpretou os resultados, escreveu o manuscrito, revisou a literatura e aprovou a versão final do manuscrito. C Giugliani: analisou e interpretou os resultados, escreveu o manuscrito, revisou a literatura e aprovou a versão final do manuscrito.

\section{Referências}

1. Victora CG, Bahl R, Barros AJD, França GVA, Horton S, Krasevec J, Murch S, Sankar MJ, Walker N, Rollins NC, Lancet Breastfeeding Series Group. Breastfeeding in the 21st century: epidemiology, mechanisms, and lifelong effect. Lancet 2016; 387(10017):475-490.

2. Chowdhury R, Sinha B, Sankar MJ, Taneja S, Bhandari N, Rollins N, Bahl R, Martines J. Breastfeeding and maternal health outcomes: a systematic review and meta-analysis. Acta Paediatr 2015; 104(467):96-113.

3. World Health Organization (WHO). Infant and young child feeding: model chapter for textbooks for medical students and allied health professionals. Genebra: WHO; 2009.

4. Boccolini CS, Boccolini PMM, Monteiro FR, Venâncio SI, Giugliani ERJ. Breastfeeding indicators trends in Brazil for three decades. Rev Saude Publica 2017; 51:108.

5. Schirm E, Schwagermann MP, Tobi H, Jong-van den Berg LTW. Drug use during breastfeeding. A survey from the Netherlands. Eur J Clin Nutr 2004; 58(2):386-390.

6. Chaves RG, Lamounier JA, César CC. Self-medication in nursing mothers and its influence on the duration of breastfeeding. J Pediatr (Rio J) 2009; 85(2):129-134.

7. Lutz BH, Bassani DG, Miranda VIA, Silveira MPT, Mengue SS, Dal Pizzol TS, Silveira MF, Bertoldi AD. Use of Medications by Breastfeeding Women in the 2015 Pelotas (Brazil) Birth Cohort Study. Int J Environ Res Public Health 2020; 17(2):568. 
8. Rowe H, Baker T, Hale TW. Maternal Medication, Drug Use, and Breastfeeding. Child Adolesc Psychiatr Clin N Am 2015; 24(1):1-20.

9. Matheson I, Kristensen K, Lunde PKM. Drug utilization in breast-feeding women. A survey in Oslo. Eur J Clin Pharmacol 1990; 38(5):453-459.

10. Paricio Talayero JM, Díaz-Gómez NM, Rivera LL, Palomares MS, Cholbi LE. Medicamentos y lactancia materna. An Pediatría Contin 2014; 12(5):239-243.

11. Sachs HC, Committee on Drugs. The Transfer of Drugs and Therapeutics Into Human Breast Milk: An Update on Selected Topics. Pediatrics 2013; 132(3):e796-e809.

12. Hale TW, Rowe HE. Medications and Mother's Milk. 16a ed. Texas: Hale Publishing; 2014.

13. Bjerrum L, Foged A. Patient information leaflets-helpful guidance or a source of confusion? Pharmacoepidemiol Drug Saf 2003; 12(1):55-59.

14. Dal Pizzol TS, Moraes CG, Diello MV, Campos PM, Pletsch JT, Giugliani C. Uso de medicamentos antidepressivos na amamentação: avaliação da conformidade das bulas com fontes bibliográficas baseadas em evidências científicas. Cad Saude Publica 2019; 35(2):e00041018.

15. Colaceci S, Giusti A, Chapin EM, Notarangelo M, De Angelis A, Vellone E, Alvaro R. The Difficulties in Antihypertensive Drug Prescription During Lactation: Is the Information Consistent? Breastfeed Med 2015; 10(10):468-473.

16. Chaves RG, Lamounier JA, César CC, Corradi MAL, Mello RP, Gontijo CM, Drumond JM. Amamentação e uso de antiinflamatórios não esteróides pela nutriz: informações científicas versus conteúdo em bulas de medicamentos comercializados no Brasil. Rev Bras Saude Materno Infant 2006; 6(3):269-276.

17. WHO Collaborating Centre. WHOCC - ATC/DDD Index [Internet]. [acessado $2018 \mathrm{dez} 6$ ]. Disponível em: https://www.whocc.no/atc_ddd_index/.

18. Agência Nacional de Vigilância Sanitária (ANVISA). Consulta a Medicamentos e Hemoderivados [Internet]. [acessado 2020 ago 12]. Disponível em: http://portal. anvisa.gov.br/medicamentos/consultas.

19. Agência Nacional de Vigilância Sanitária (ANVISA). Bulário Eletrônico [Internet]. [acessado 2016 out 11]. Disponível em: http://portal.anvisa.gov.br/bulario -eletronico1.

20. Agência Nacional de Vigilância Sanitária (ANVISA). Resolução - RDC n 47, de 8 de setembro de 2009. Estabelece regras para elaboração, harmonização, atualização, publicação e disponibilização de bulas de medicamentos para pacientes e para profissionais de saúde. Diário Oficial da União; 2009.

21. Brasil. Ministério da Saúde (MS). Amamentação e uso de medicamentos e outras substâncias. $2^{\mathrm{a}}$ ed. Brasília: MS; 2010.

22. U.S. National Library of Medicine. Drugs and Lactation Database (LactMed) [Internet]. [acessado 2018 nov 13]. Disponível em: https://toxnet.nlm.nih.gov/ newtoxnet/lactmed.htm.

23. Wolters Kluwer. Search - UpToDate [Internet]. [acessado 2018 nov 12]. Disponível em: https://www.uptodate.com/contents/search.
24. IBM Corporation. Drug Result Page - MICROMEDEX [Internet]. [acessado 2018 nov 12]. Disponível em: https://www.micromedexsolutions.com/micromedex $2 /$ librarian/PFDefaultActionId/evidencexpert.DoIntegratedSearch\#.

25. Akus M, Bartick M. Lactation Safety Recommendations and Reliability Compared in 10 Medication Resources. Ann Pharmacother 2007; 41(9):1352-1360.

26. Landis JR, Koch GG. The measurement of observer agreement for categorical data. Biometrics 1977; 33(1):159-174

27. Arguello B, Salgado TM, Fernandez-Llimos F. Assessing the information in the Summaries of Product Characteristics for the use of medicines in pregnancy and lactation. Br J Clin Pharmacol 2015; 79(3):537544.

28. Davanzo R, Copertino M, De Cunto A, Minen F, Amaddeo A. Antidepressant Drugs and Breastfeeding: A Review of the Literature. Breastfeed Med 2011; 6(2):89-98.

29. Schüler-Faccini L, Sanseverino MTV, Giugliani C. Medicamentos e Outras Exposições na Gestação e na Amamentação. In: Medicina Ambulatorial: Condutas de Atenção Primária Baseadas em Evidências. $4^{a}$ ed. Porto Alegre: Artmed; 2013.

30. Caldeira TR, Neves ERZ, Perini E. Historical evolution of package inserts in Brazil. Cad Saude Publica 2008; 24(4):737-743.

31. Cruz F, Caldeira T, Rediguieri C. Bulas e Rótulos. In: A regulação de medicamentos no Brasil. Porto Alegre: Artmed; 2013.

32. European Medicines Agency (EMEA). Guideline on risk assessment of medicinal products on human reproduction and lactation: from data to labelling [Internet]. EMEA; 2008 [acessado 2020 ago 12]. Disponível em: https://www.ema.europa.eu/en/risk-assessment-medicinal-products-human-reproduction-lactation-data-labelling\#current-effective-version-section.

Artigo apresentado em 09/10/2019

Aprovado em 25/08/2020

Versão final apresentada em 27/08/2020

Editores-chefes: Romeu Gomes, Antônio Augusto Moura da Silva 